\title{
Sedimentation of a cylindrical particle in a Carreau fluid
}

\author{
Jyh-Ping Hsu ${ }^{a, *}$, Ching-Feng Shie ${ }^{a}$, Shiojenn Tseng ${ }^{\mathrm{b}}$ \\ a Department of Chemical Engineering, National Taiwan University, Taipei 10617, Taiwan \\ ${ }^{\mathrm{b}}$ Department of Mathematics, Tamkang University, Tamsui, Taipei 25137, Taiwan
}

Received 11 October 2004; accepted 19 January 2005

Available online 10 March 2005

\begin{abstract}
The drag coefficient of an isolated, rigid cylindrical particle in a Carreau fluid is evaluated. The result of numerical simulation reveals that, in general, the shear-thinning nature of a Carreau fluid yields a drag coefficient smaller than that for the corresponding Newtonian fluid. Also, the smaller the Reynolds number, the more appreciable the decrease of the drag coefficient as the relaxation time constant of the Carreau fluid increases. The influence of the index parameter of a Carreau fluid on the drag coefficient depends largely on the magnitude of the relaxation time constant and is insensitive to the Reynolds number. Only if the relaxation time constant is sufficiently large is the influence of the index parameter on the drag coefficient significant. If the Reynolds number and/or the relaxation time constant is sufficiently large, the flow field upstream of a particle becomes asymmetric to that downstream. In general, the influence of the index parameter, the relaxation time constant, and the Reynolds number on the flow field follows the order index parameter $<$ relaxation time constant $<$ Reynolds number.
\end{abstract}

(c) 2005 Elsevier Inc. All rights reserved.

Keywords: Sedimentation; Cylindrical particle; Carreau fluid; Drag coefficient

\section{Introduction}

The terminal velocity of particles in a fluid medium is one of its basic characteristics. Through appropriate measurements, the drag acting on a particle can be estimated, which, in turn, can be used to evaluate its physical properties. Practical application includes, for example, design of viscometers, fluidized beds, and pipe transportation systems. The wide applications of terminal velocity have triggered active studies in the past; various theoretical and experimental attempts have been made to investigate the sedimentation behavior of particles. Available results for the terminal velocity and the drag for the free setting of particles in Newtonian fluids are ample in the literature. In contrast, those in non-Newtonian fluids are relatively limited.

Fluids that exhibit non-Newtonian behavior are not uncommon in practice. Blood, polymer solution, and emulsion are typical examples. Many empirical expressions have been

\footnotetext{
* Corresponding author. Fax: +886-2-23623040.

E-mail address: jphsu@ntu.edu.tw (J.-P. Hsu).
}

proposed for the description of these fluids [1]. For sedimentation in a non-Newtonian fluid, almost all of the available results are limited to spherical particles [2-5] and that for nonspherical particles are relatively limited [6-11]. Several attempts were made concerning the sedimentation in a viscoelastic fluid, and behaviors that are different from those in a Newtonian fluid were observed (e.g., [12-16]). The sedimentation of particles in a shear-thinning fluid has also been studied by many investigators. Under the creeping flow condition, Rodrigue et al. [16] derived the drag coefficients of both rigid spheres and bubbles in an infinite Carreau fluid. It was shown that the influence of the shear-thinning nature of a Carreau fluid on a rigid sphere is greater than that on a bubble. Turian [17] and Uhlherr et al. [18] investigated experimentally the sedimentation of a rigid sphere in a cylindrical tube filled with an inelastic power-law fluid. Navez and Walters [19] analyzed the settling of a sphere in shear-thinning polymer solutions. They pointed out that the influence of the shear-thinning nature of a fluid on the drag coefficient is greater than that of the elastic nature of the fluid. In a study of the wall effect on the motion of a particle 
under creeping flow condition Missirlis et al. [20] evaluated the drag coefficient for the case when a rigid sphere is in a cylinder filled with a power-law fluid. Ceylan et al. [21] proposed a theoretical model for the estimation of the drag force on a rigid sphere in a power-law fluid. The applicability of the model proposed was justified by experimental observations [22], and it was found that it is applicable for Reynolds number up to 1000. Machač et al. [23] analyzed the terminal falling velocity of spherical particles in a Carreau fluid under creeping flow conditions. Blackery and Mitsoulis [24] simulated the sedimentation of a rigid sphere in a cylinder filled with Bingham plastic fluid under creeping flow condition. Pazwash and Robertson [25] measured the force acting on a disk in Bingham fluids. Torrest [26] claimed that Stokes's law is capable of describing the settling of gravel chips in viscous non-Newtonian hydroxyethyl cellulose polymer solutions. Peden and Luo [27] reported the settling velocity of cylindrical and disk-shaped particles in drilling and fracturing fluids. Reynolds and Jones [28] measured the drag forces on various types of particles in non-Newtonian fluids. They concluded that using a volume-equivalent diameter is most appropriate in correlating their experimental data. Chhabra [29] examined the settling of a cylindrical particle in a cylinder filled with power-law liquids. Cho et al. [30] studied the sedimentation of a thin cylinder in both Newtonian and nonNewtonian fluids. General discussions on the subject are also available [31-33].

In this study, the sedimentation of a rigid cylindrical particle in an infinite Carreau fluid is investigated for the case of a low to medium-large Reynolds number. In this case, since the governing equations for the flow filed are nonlinear, solving them analytically becomes nontrivial. Here, a finite element scheme is adopted to circumvent the difficulty encountered. The influences of the key parameters of the problem under consideration, including the aspect ratio of a particle, the magnitude of the Reynolds number, and the nature of the fluid, on the drag coefficient are discussed.

\section{Theory}

Referring to Fig. 1, we consider the sedimentation of an isolated, rigid cylindrical particle of diameter $d$ and height $h$ in a Carreau fluid. For convenience, the particle is held fixed and the surrounding fluid moves with bulk velocity $u_{t}$, the terminal velocity of the particle. The cylindrical coordinates are adopted with their origin located at the center of the particle; $r$ and $z$ are the radial and axial coordinates. At steady state, the flow field can be described by

$$
\begin{aligned}
& \rho \mathbf{u} \cdot \nabla \mathbf{u}=-\nabla P+\nabla \cdot \boldsymbol{\tau}, \\
& \nabla \cdot \mathbf{u}=0,
\end{aligned}
$$

where $\rho$ is the density of fluid, $P$ is the pressure, $\nabla$ is the gradient operator, $\boldsymbol{\tau}$ is the stress tensor, and $\mathbf{u}$ is the fluid velocity. The constitutive equation for a generalized New-

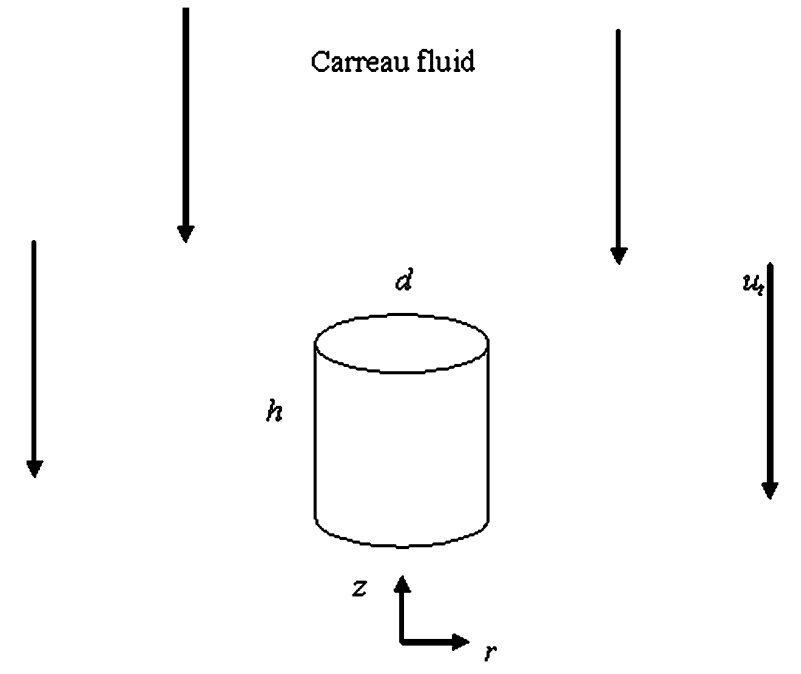

Fig. 1. Sedimentation of a rigid, cylindrical particle of diameter $d$ and height $h$ in an infinite Carreau fluid; $u_{t}$ is the terminal velocity of the particle. In mathematical modeling, the particle is held fixed and the fluid moves with bulk velocity $u_{t} . r$ and $z$ are the radial and the axial coordinates.

tonian fluid can be expressed as

$\boldsymbol{\tau}=-\eta(\dot{\gamma}) \dot{\gamma}=-\eta(\dot{\gamma})\left(\nabla \mathbf{u}+(\nabla \mathbf{u})^{T}\right)$,

where $\dot{\gamma}$ and $\dot{\gamma}=[(\dot{\gamma}: \dot{\gamma}) / 2]^{1 / 2}$ are respectively the rate of strain tensor and its strength, $\eta$ is the apparent viscosity, and the superscript $T$ represents matrix transpose. Carreau proposed using [1]

$\eta(\dot{\gamma})=\eta_{\infty}+\left(\eta_{0}-\eta_{\infty}\right)\left[1+(\lambda \dot{\gamma})^{2}\right]^{(n-1) / 2}$,

where $\eta_{0}$ and $\eta_{\infty}$ are respectively the apparent viscosities corresponding to the minimum and the maximum $\dot{\gamma}, \lambda$ is the relaxation time constant of the Carreau fluid, and $n$ is its index parameter. Under conditions of practical significance, $\eta_{0} \gg \eta_{\infty}$, and therefore, Eq. (4) reduces to

$\eta(\dot{\gamma})=\eta_{0}\left[1+(\lambda \dot{\gamma})^{2}\right]^{(n-1) / 2}$.

Note that if $n \rightarrow 1$ and/or $\lambda \rightarrow 0$, the corresponding fluid is Newtonian.

The boundary conditions associated with Eqs. (1) and (2) are assumed to be

$u_{z}=u_{t} \quad$ as $r \rightarrow \infty$ or $z \rightarrow \infty$,

$u_{z}=0$ on particle surface,

where $u_{z}$ is the $z$-component of fluid velocity. The symmetric nature of the present problem also requires that

$\frac{\partial \mathbf{u}}{\partial r}=0, \quad r=0$.

For the present case, the drag force acting on a particle, $F_{\mathrm{D}}$, can be expressed as

$F_{\mathrm{D}}=C_{\mathrm{D}} \frac{\pi d^{2}}{4} \frac{\rho u_{z}^{2}}{2}$, 
where $C_{\mathrm{D}}$ is the drag coefficient. For a general particle in a Carreau fluid, $C_{\mathrm{D}}$ is related to the shape and the orientation of the particle and the nature of the fluid. The exact analytical expression for $C_{\mathrm{D}}$ has not been reported. Usually, an empirical relation is assumed with its adjustable parameters estimated from experimental observations [34]. In the present study, the governing equations, Eqs. (1) and (2), and the associated boundary conditions, Eqs. (6)-(8), are solved numerically by FIDAP, a finite element scheme based on the Galerkin method. The drag force $F_{\mathrm{D}}$ was calculated by FIDAP, and the drag coefficient $C_{\mathrm{D}}$ is obtained by substituting the calculated $F_{\mathrm{D}}$ into Eq. (9).

\section{Results and discussion}

The applicability of the present approach is examined by comparing the result obtained with that predicted by the semiempirical expression proposed by Machač et al. [34], which is limited to low Reynolds numbers $\left(\operatorname{Re}_{0}<0.1\right)$. Fig. 2 shows the typical variation of $C_{\mathrm{D}}$ as a function of $\mathrm{Re}_{0}$. As can be seen from this figure, if $\operatorname{Re}_{0}<0.1$, the result based on the present approach agrees well with that predicted by Machač et al. [34]. On the other hand, if $\operatorname{Re}_{0}$ exceeds about 0.1 , the deviation of the latter from the former becomes appreciable. The deviation is positive and increases with $\operatorname{Re}_{0}$. This is expected because the inertial effect, which is significant when $\operatorname{Re}_{0}$ becomes large, is neglected in Machač et al. [34]. In general, the performance of the present approach is satisfactory. In subsequent discussions, the influences of the key parameters of the problem under consideration on the drag coefficient $C_{\mathrm{D}}$ and on the flow field are investigated through numerical simulation.

Fig. 3 illustrates the variation of $C_{\mathrm{D}}$ as a function of $\operatorname{Re}_{0}$ and $\mathrm{Cu}$. This figure reveals that, in general, $C_{\mathrm{D}}$ decreases with the increase in both $\mathrm{Cu}$ and $\mathrm{Re}_{0}$. The former arises from the fact that the larger the $\mathrm{Cu}$ the more important the shear-thinning nature of a fluid is, and the latter is similar to that for the case of a Newtonian fluid. If $\mathrm{Re}_{0}$ is sufficiently small, $\log \left(C_{\mathrm{D}}\right)$ varies linearly with $\log \left(\mathrm{Re}_{0}\right)$ for both the present Carreau fluid and the corresponding Newtonian fluid. The latter is expected because Stokes's law is applicable if $\operatorname{Re}_{0}$ is small. For both the present Carreau fluid and the corresponding Newtonian fluid, a positive deviation from the Stokes's law relation is observed if $\operatorname{Re}_{0}$ is sufficiently large. It is found that the smaller the $\operatorname{Re}_{0}$ the more appreciable the decrease of $C_{\mathrm{D}}$ as $\mathrm{Cu}$ increases. For example, if $n=0.6$, the decreases in $C_{\mathrm{D}}$ as $\mathrm{Cu}$ varies from 0.1 to 100 are respectively $70.81,70.72$, and $65.56 \%$ for $\operatorname{Re}_{0}$ equals $0.1,1$, and 10 . Further calculations reveal that the influence of $\mathrm{Cu}$ on $C_{\mathrm{D}}$ is more significant at a smaller $n$ than that at a larger $n$, which is expected since the smaller the $n$ the more significant the shear thinning nature of a Carreau fluid is. For example, if $\mathrm{Re}_{0}=0.1$, the decreases in $C_{\mathrm{D}}$ as $\mathrm{Cu}$ varies from 0.1 to 100 are $84.16,70.81$, and $42.36 \%$ for $n$ equal to $0.4,0.6$, and 0.8 , respectively.

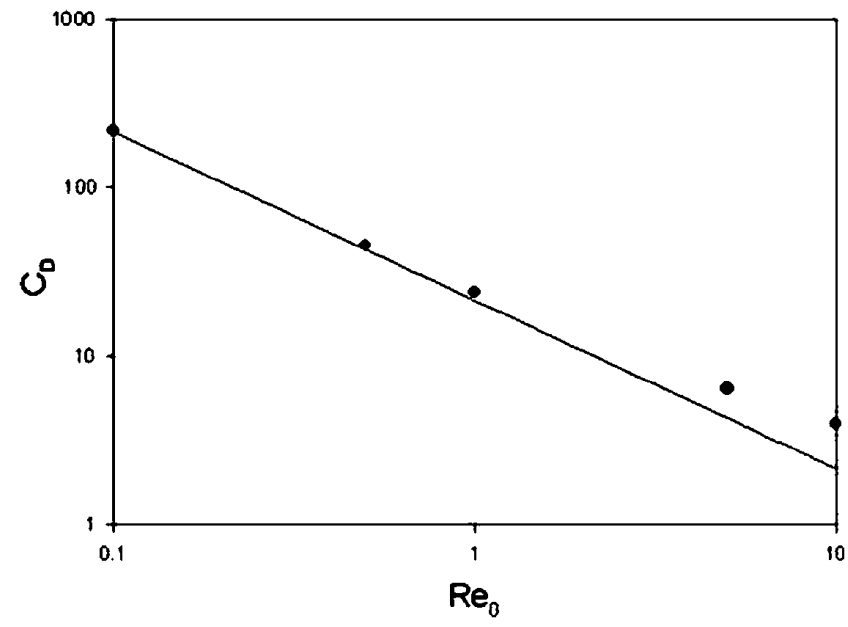

Fig. 2. Variation of $C_{\mathrm{D}}$ as a function of $\mathrm{Re}_{0}$ for the case of a short cylinder in a Carreau fluid for the case when $b=h / d=0.5, \mathrm{Cu}=0.1$, and $n=0.6$. Solid line, semiempirical result of Machač et al. [34]; discrete symbols, numerical result.

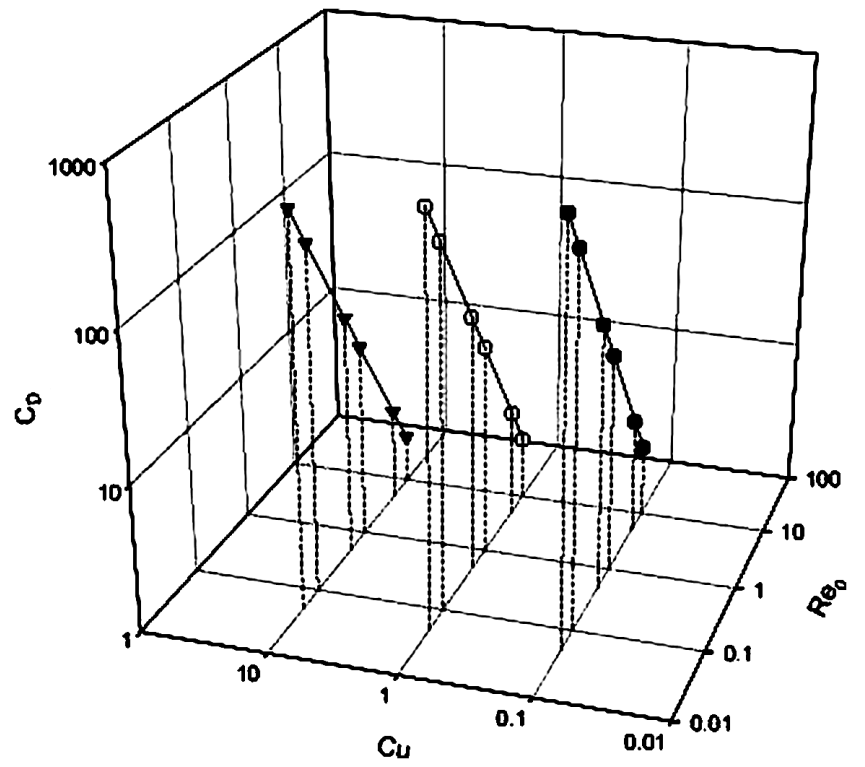

Fig. 3. Variation of $C_{\mathrm{D}}$ as a function of $\mathrm{Cu}$ and $\mathrm{Re}_{0}$ at various $\mathrm{Cu}$ for the case when $b=h / d=0.5$ and $n=0.6$.

Fig. 4 illustrates the simulated variations of the drag coefficient $C_{\mathrm{D}}$ as a function of $n$ at various combinations of $\mathrm{Cu}$ and $\mathrm{Re}_{0}$. In general, $C_{\mathrm{D}}$ increases with the increases in $n$, which is expected because the larger the $n$ the less significant the shear-thinning effect of a fluid is. Fig. 4 indicates that if $\mathrm{Cu}$ is small, regardless of the value of $\mathrm{Re}_{0}$, the influence of $n$ on $C_{\mathrm{D}}$ is inappreciable. For example, if $\mathrm{Cu}=1$, the increases in $C_{\mathrm{D}}$ as $n$ increases from 0.2 to 1.0 are 2.16, 4.20, and $11.46 \%$ for $\operatorname{Re}_{0}=0.1,1$, and 10 , respectively. On the other hand, if $\mathrm{Cu}$ is large, $C_{\mathrm{D}}$ varies appreciably as $n$ increases, even if $\mathrm{Re}_{0}$ is small. For instance, if $\mathrm{Cu}=10$, the increases in $C_{\mathrm{D}}$ as $n$ increases from 0.2 to 1.0 are respectively $176.93,178.20$, and $213.03 \%$ for $\operatorname{Re}_{0}=0.1,1$, and 10 . These imply that, the influence of $n$ on $C_{\mathrm{D}}$ depends largely on the magnitude of $\mathrm{Cu}$ and is insensitive to the variation in 


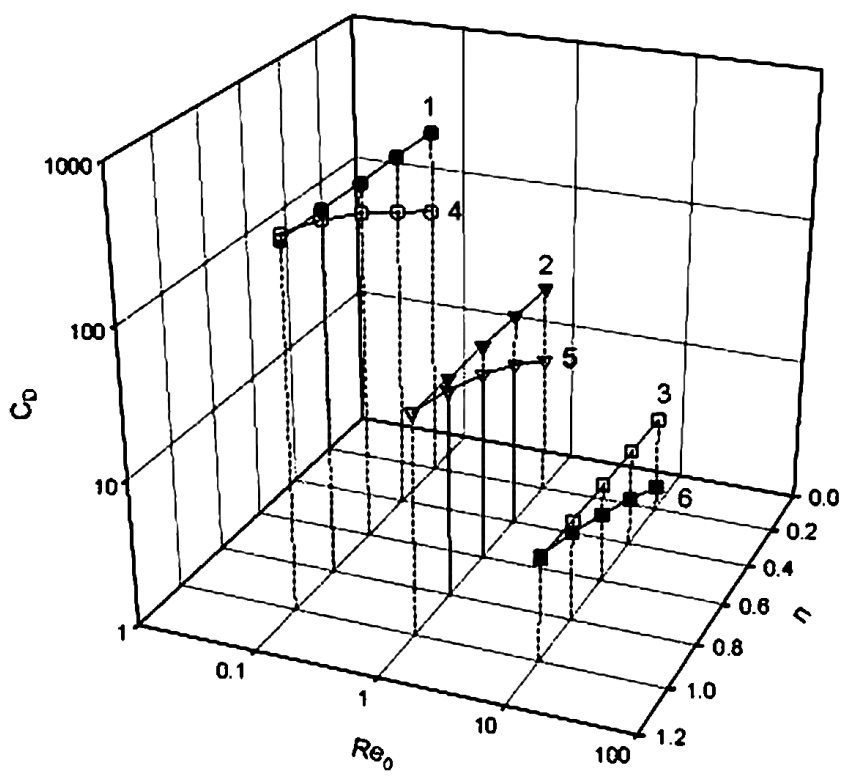

Fig. 4. Variation of $C_{\mathrm{D}}$ as a function of $n$ at various combinations of $\mathrm{Cu}$ and $\operatorname{Re}_{0}$ for the case when $b=h / d=0.5$ and $n=0.6$. Curves $1-3, \mathrm{Cu}=1$; $4-6, \mathrm{Cu}=10$. Curves 1 and $4, \operatorname{Re}_{0}=0.1 ; 2$ and $5, \operatorname{Re}_{0}=1 ; 3$ and 6 , $\operatorname{Re}_{0}=10$.

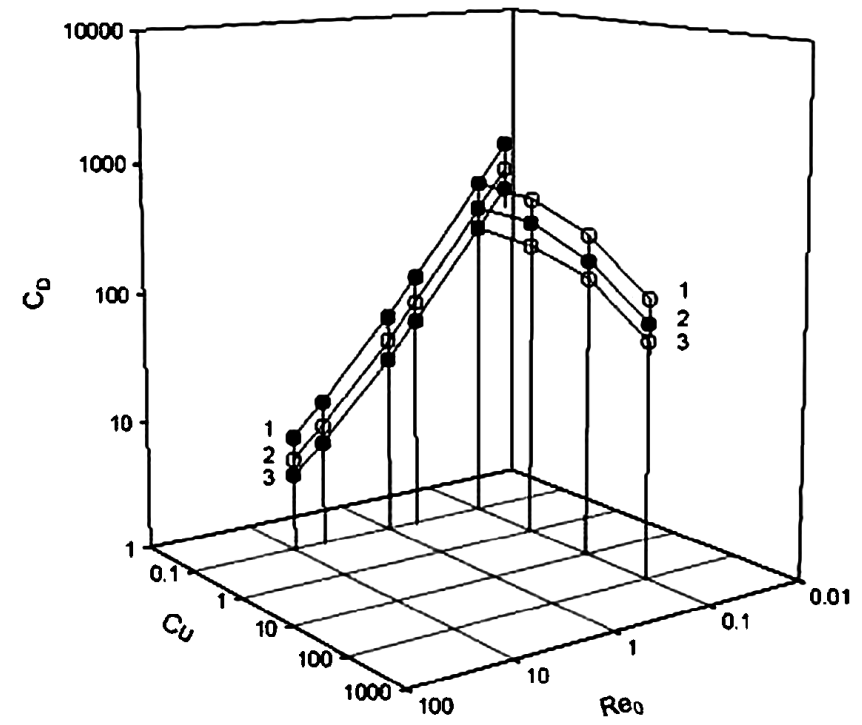

Fig. 5. Variation of $C_{\mathrm{D}}$ as a function of $\mathrm{Cu}$ and $\mathrm{Re}_{0}$ at various $b(=h / d)$ for the case when $n=0.6$ and $\mathrm{Cu}=0.1$. Curve $1, b=2 ; 2, b=1 ; 3, b=0.5$.

$\mathrm{Re}_{0}$. Only if $\mathrm{Cu}$ is sufficiently large is the influence of $n$ on $C_{\mathrm{D}}$ important. Fig. 4 also indicates that for the same $\mathrm{Re}_{0}$, the $C_{\mathrm{D}}$ for a fluid with a large $\mathrm{Cu}$ approaches that with a small $\mathrm{Cu}$ as $n$ approaches unity, and it is possible that the former exceeds the latter. This is because the shear-thinning nature of the present Carreau fluid is governed by both $\lambda$ and $n$.

Fig. 5 shows the variation of the drag coefficient $C_{\mathrm{D}}$ as a function of $\mathrm{Cu}$ and Reynolds number $\mathrm{Re}_{0}$ at various particle aspect ratios $b(=h / d)$. That as a function of $n$ at various $b$ is present in Fig. 6. In Fig. 5, because the cross-sectional area (or $d$ ) of a particle is fixed, the larger the $b$, the larger its lateral surface, the greater the drag exerted on the particle,

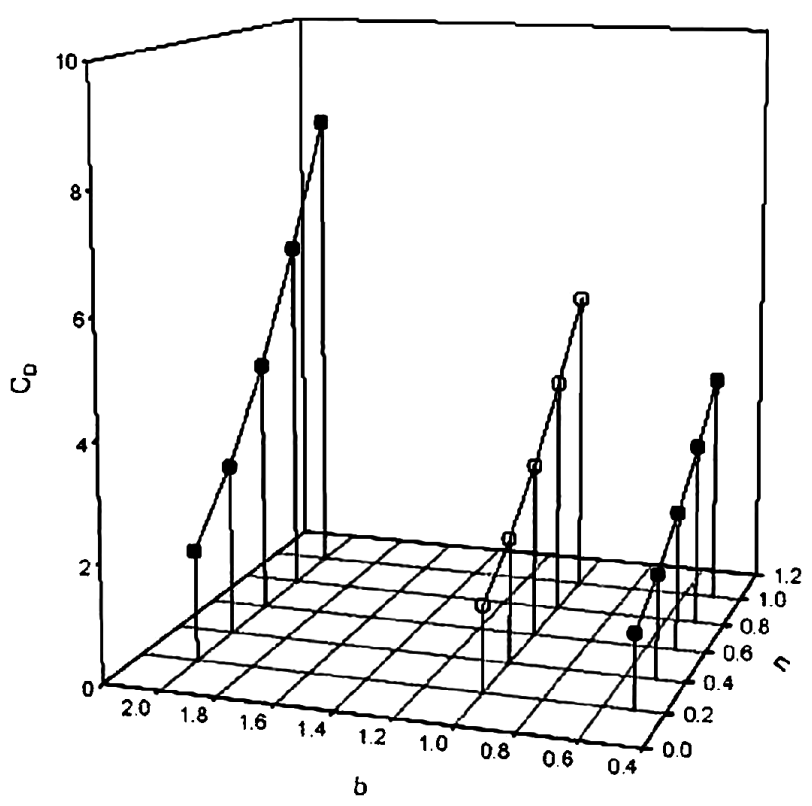

Fig. 6. Variation of $C_{\mathrm{D}}$ as a function of $n$ at various $b(=h / d)$ for the case when $n=0.6, \mathrm{Cu}=10$, and $\operatorname{Re}_{0}=10$.

and therefore, a larger $C_{\mathrm{D}}$. Fig. 5 suggests that $\log \left(C_{\mathrm{D}}\right)$ various nonlinearly with $\log (\mathrm{Cu})$. For a fixed $b, C_{\mathrm{D}}$ decreases with the increase in $\mathrm{Cu}$, and for a fixed $\mathrm{Cu}, C_{\mathrm{D}}$ decreases with the increase in $b$. The former arises from the fact that the larger $\mathrm{Cu}$ the more significant the shear-thinning effect is, and the latter is consistent with the previous discussion about the effect of $b$. The general trend observed in Fig. 6 can be explained by similar reasoning.

The influences of $\mathrm{Re}_{0}, \mathrm{Cu}$, and $n$ on the flow field are illustrated in Figs. 7-12. Figs. $7 \mathrm{a}$ and $7 \mathrm{~b}$ indicate that if $\mathrm{Re}_{0}$ is small $(<0.1)$, the flow field in the upstream of a particle is symmetric to that of its downstream. It becomes asymmetric, however, if $\operatorname{Re}_{0}$ is large $(>0.1)$, as can be seen in Figs. 7c-7f. Here, because $\operatorname{Re}_{0}$ is not very large and the liquid phase is infinitely large, the deformation of the flow field due to the presence of the particle is inappreciable. It is clearer by examining the variation of the vorticity shown in Fig. 8, where the contours become asymmetric if $\mathrm{Re}_{0}$ exceeds about 0.1 . The flow field also becomes asymmetric if $\mathrm{Cu}$ is large, as suggested by the variation in fluid velocity illustrated in Fig. 9. This behavior arises from the shearthinning nature of the Carreau fluid considered, since the larger the $\mathrm{Cu}$ the less viscous the fluid is, and it is easier for the fluid to separate from the particle surface in the downstream. Figs. 10-12 reveal that the influence of $n$ on the flow field is not as appreciable as that of $\mathrm{Cu}$ and that of $\mathrm{Re}_{0}$. In general, the influence of $n, \mathrm{Cu}$, and $\mathrm{Re}_{0}$ on the flow field follows the order $n<\mathrm{Cu}<\mathrm{Re}_{0}$.

\section{Conclusion}

In summary, the sedimentation of an isolated cylindrical particle in a Carreau fluid is analyzed by evaluating the 


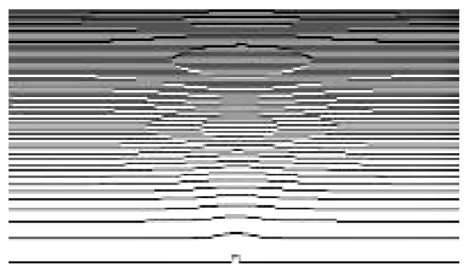

(a)

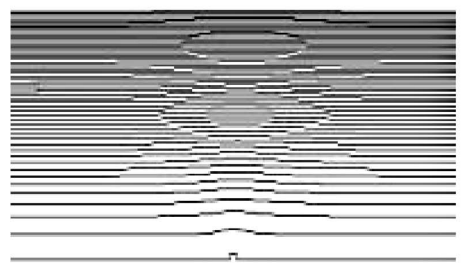

(b)

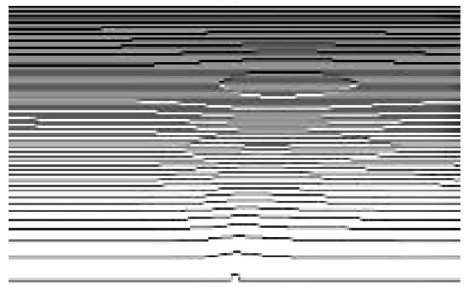

(c)

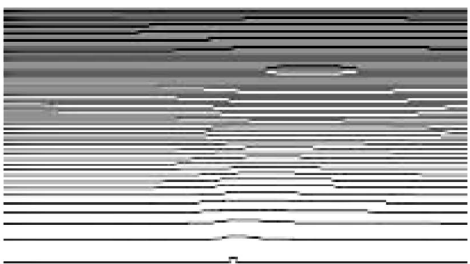

(d)

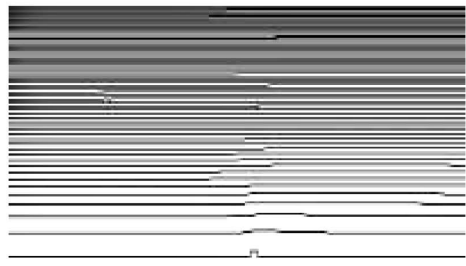

(e)

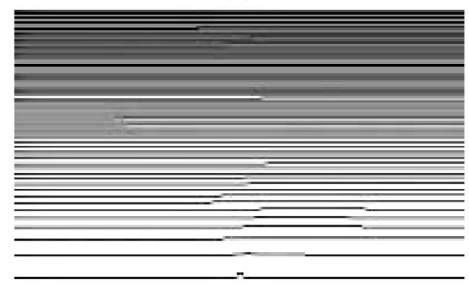

(f)

Fig. 7. Streamline contours at various $\operatorname{Re}_{0}$ for the case when $b=h / d=0.5$ and $\mathrm{Cu}=1$. (a) $\operatorname{Re}_{0}=0.05$, (b) $\operatorname{Re}_{0}=0.1$, (c) $\operatorname{Re}_{0}=0.5$, (d) $\operatorname{Re}_{0}=1$, (e) $\operatorname{Re}_{0}=5$, (f) $\operatorname{Re}_{0}=10$.

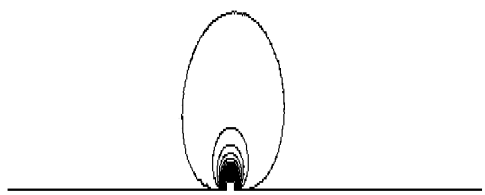

(a)

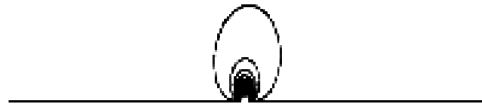

(b)

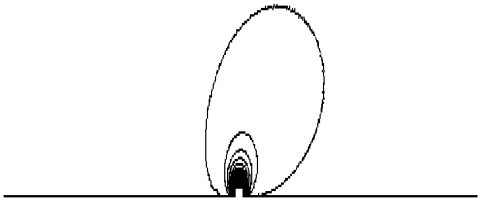

(c)

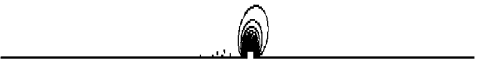

(d)

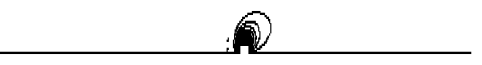

(e)

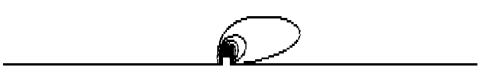

(f)

Fig. 8. Vorticity contours at various $\mathrm{Re}_{0}$ for the case of Fig. 7.

drag coefficient under various conditions. We show that, in general, the qualitative behavior of the variation of the drag coefficient as a function of Reynolds number is similar to that of a Newtonian fluid, that is, if Reynolds number is sufficiently small, a Stokes's-law-like relation between drag coefficient and Reynolds number exists, and a positive de- 


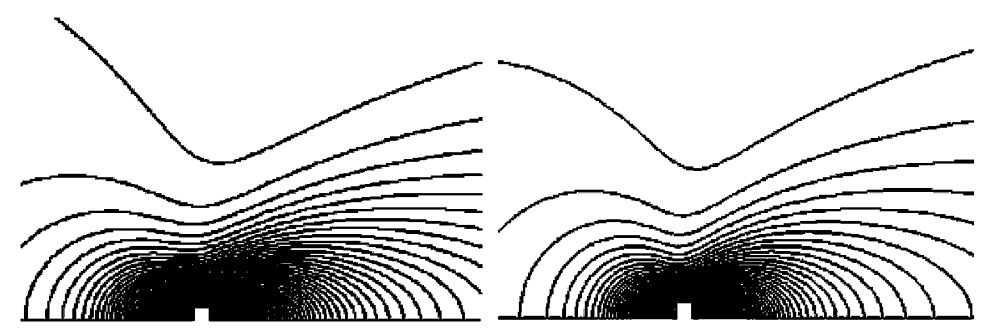

(a)

(c)

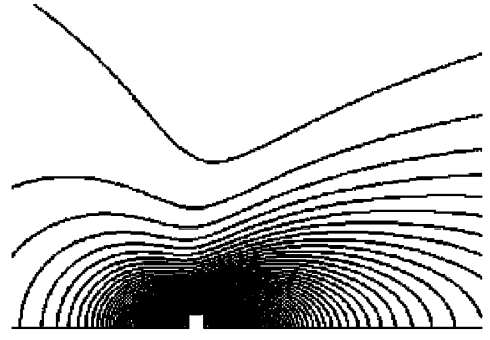

(b)

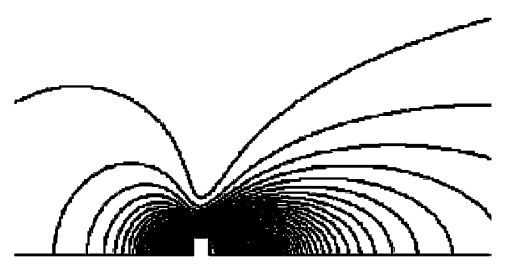

(d)

Fig. 9. Velocity contours at various $\mathrm{Cu}$ for the case when $b=h / d=0.5$ and $\mathrm{Re}_{0}=1$. (a) $\mathrm{Cu}=0.1$, (b) $\mathrm{Cu}=1$, (c) $\mathrm{Cu}=10,(\mathrm{~d}) \mathrm{Cu}=100$.

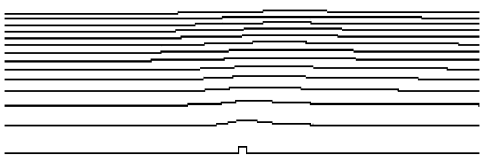

(a)

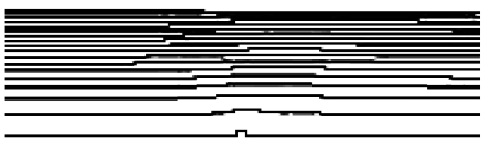

(b)

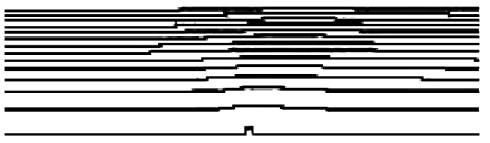

(c)

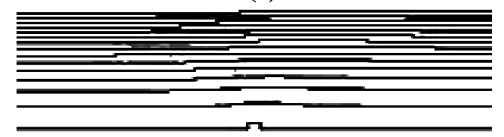

(d)

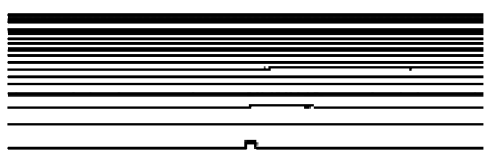

(e)

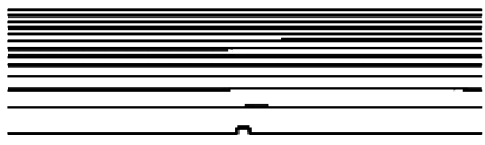

(f)

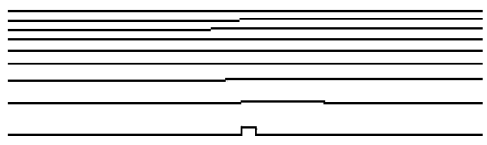

(g)

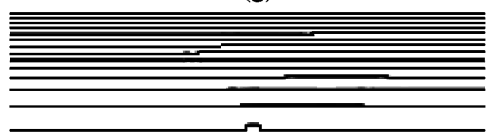

(h)

Fig. 10. Streamline contours at different combinations of $\operatorname{Re}_{0}$ and $\mathrm{Cu}$ for the case when $b=h / d=0.5$. (a)-(d) $\operatorname{Re}_{0}=1$ and $\mathrm{Cu}=1 ;(\mathrm{e})-(\mathrm{h}) \mathrm{Re}_{0}=10$ and $\mathrm{Cu}=10$. (a) $n=0.2$, (b) $n=0.4$, (c) $n=0.6$, (d) $n=0.8$, (e) $n=0.2$, (f) $n=0.4$, (g) $n=0.6$, (h) $n=0.8$.

viation from that law is observed when Reynolds number becomes large. If the relaxation time constant of the Carreau fluid is small, regardless of the value of Reynolds number, the influence of the index parameter of the Carreau fluid on the drag coefficient is inappreciable. On the other hand, if the relaxation time constant is large, $C_{D}$ varies appreciably as $n$ increases, even if the Reynolds number is small. These imply that the influence of the index parameter on the drag coefficient depends largely on the magnitude of the relaxation time constant, and is insensitive to the variation in Reynolds number. For the same Reynolds number, the drag coefficient for a fluid with a large relaxation time constant approaches that with a small relaxation time constant as the index parameter approaches unity, that is, Newtonian fluid, and it is possible that the former exceeds the latter. As in the case of Newtonian fluids, if the Reynolds number is small, the flow field in the upstream of a particle is symmetric to that of its downstream. It becomes asymmetric, however, if the Reynolds number is large, where boundary separation may occur. The flow field also becomes asymmetric if the relaxation time constant becomes large. The influence of the index parameter on the flow field is not as appreciable as that of the relaxation time constant and that of the Reynolds number. 


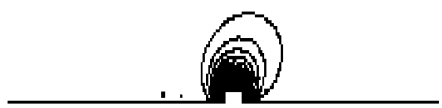

(a)

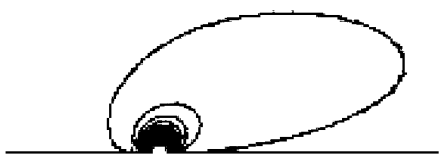

(b)

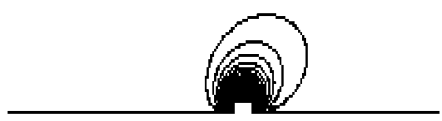

(c)

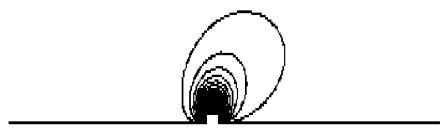

(d)

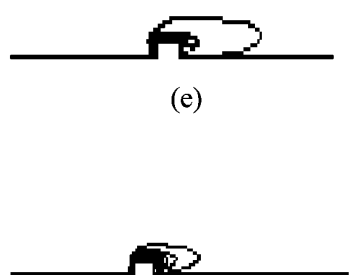

(f)

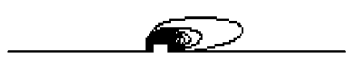

(g)

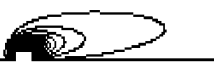

(h)

Fig. 11. Vorticity contours at different combinations of $\mathrm{Re}_{0}$ and $\mathrm{Cu}$ for the case of Fig. 10.

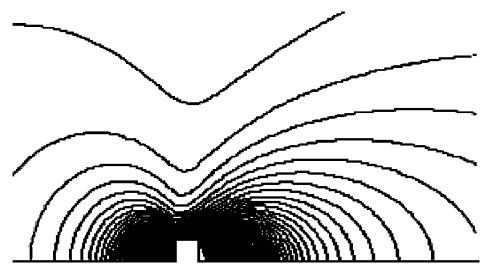

(a)

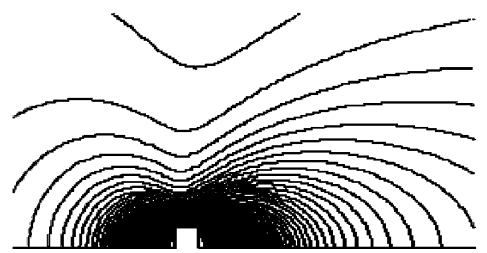

(b)

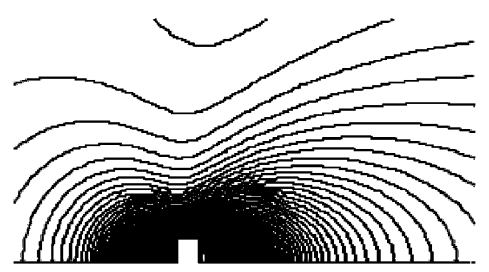

(c)

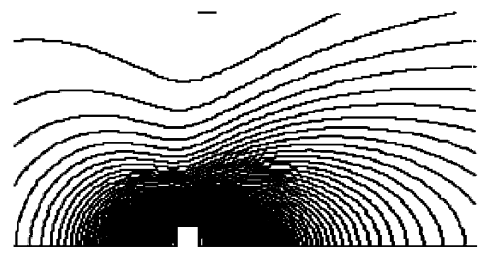

(d)

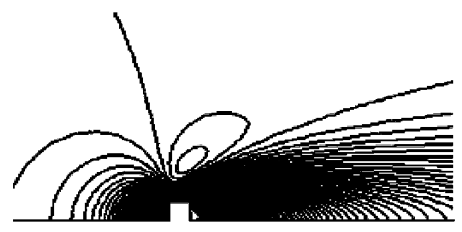

(e)

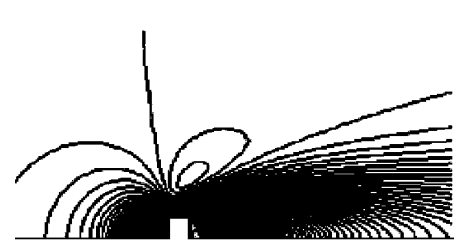

(f)

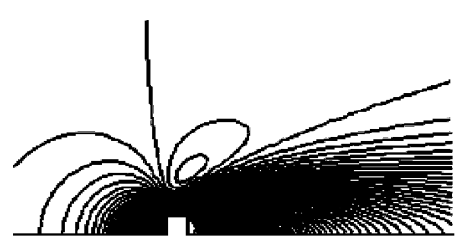

(g)

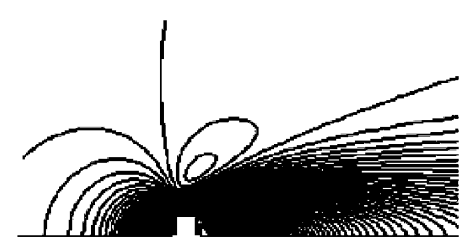

(h)

Fig. 12. Velocity contours at different combinations of $\operatorname{Re}_{0}$ and $\mathrm{Cu}$ for the case when $b=h / d=0.5$. (a)-(d) $\operatorname{Re}_{0}=1$ and $\mathrm{Cu}=10$; (e)-(h) $\operatorname{Re}_{0}=10$ and $\mathrm{Cu}=1$. (a) $n=0.2$, (b) $n=0.4$, (c) $n=0.6$, (d) $n=0.8$, (e) $n=0.2$, (f) $n=0.4$, (g) $n=0.6$, (h) $n=0.8$. 


\section{Acknowledgment}

This work is supported by the National Science Council of the Republic of China.

\section{References}

[1] R.B. Bird, W.E. Stewart, E.N. Lightfoot, Transport Phenomena, second ed., Wiley, New York, 2002.

[2] R.P. Chhabra, C. Tiu, P.H.T. Uhlherr, Can. J. Chem. Eng. 59 (1981) 771-775.

[3] R.P. Chhabra, P.H.T. Uhlherr, Can. J. Chem. Eng. 58 (1980) 124-128.

[4] B. Mena, O. Manero, L.G. Leal, J. Non-Newtonian Fluid Mech. 26 (1987) 247-275.

[5] C.K. Vassilios, Chem. Eng. Sci. 59 (2004) 4437-4447.

[6] R.P. Chhabra, K. Rami, P.H.T. Uhlherr, Chem. Eng. Sci. 56 (2001) 2221-2227.

[7] D. Rodrigue, R.P. Chhabra, D. DeKee, Can. J. Chem. Eng. 72 (1994) 588-593.

[8] Q. Xu, E.E. Michaelides, Int. J. Numer. Methods Fluids 22 (1996) $1075-1087$.

[9] A. Tripathi, R.P. Chhabra, T. Sundararajan, Ind. Eng. Chem. Res. 33 (1994) 403-410.

[10] A. Tripathi, R.P. Chhabra, Am. Inst. Chem. Eng. J. 41 (1995) 728-731.

[11] Y.J. Liu, D.D. Joseph, J. Fluid Mech. 255 (1993) 255-595.

[12] M.B. Bush, J. Non-Newtonian Fluid Mech. 55 (1994) 229-247.

[13] J. Feng, D.D. Joseph, R. Glowinski, T.W. Pan, J. Fluid Mech. 283 (1995) 1-16.

[14] P.Y. Huang, J. Feng, D.D. Joseph, J. Fluid Mech. 271 (1994) 1-16.

[15] P.Y. Huang, H.H. Hu, D.D. Joseph, J. Fluid Mech. 362 (1998) 297325.
[16] D. Rodrigue, D. DeKee, C.F. Chan Man Fong, Chem. Eng. Commun. 154 (1996) 203-215.

[17] R.M. Turian, Am. Inst. Chem. Eng. J. 13 (1967) 999-1006.

[18] P.H.T. Uhlherr, T.N. Le, C. Tiu, Can. J. Chem. Eng. 54 (1976) 497502.

[19] V. Navez, K. Walters, J. Non-Newtonian Fluid Mech. 67 (1996) 325334.

[20] K.A. Missirlis, D. Assimacopoulos, E. Mitsoulis, R.P. Chhabra, J. Non-Newtonian Fluid Mech. 96 (2001) 459-471.

[21] K. Ceylan, S. Herdem, T. Abbasov, Powder Technol. 103 (1999) 286291.

[22] A.M. Lali, A.S. Khare, J.B. Joshi, Powder Technol. 57 (1989) 3950.

[23] I. Machač, B. Šiška, L. Machačcvá, Chem. Eng. Proc. 39 (2000) 365-369.

[24] J. Blackery, E. Mitsoulis, J. Non-Newtonian Fluid Mech. 70 (1997) 59-77.

[25] H. Pazwash, J.M. Robertson, J. Hydraul. Res. 13 (1975) 35-55.

[26] R.S. Torrest, Am. Inst. Chem. Eng. J. 29 (1983) 506-508.

[27] J.M. Peden, Y. Luo, SPE Drilling Eng. 2 (1987) 337-343.

[28] P.A. Reynolds, T.E.R. Jones, Int. J. Miner. Process. 25 (1989) $47-$ 77.

[29] R.P. Chhabra, Can. J. Chem. Eng. 70 (1992) 385-386.

[30] K. Cho, Y.I. Cho, N.A. Park, J. Non-Newtonian Fluid Mech. 45 (1992) 105-145.

[31] R. Clift, J. Grace, M.E. Weber, Bubbles, Drops and Particles, Academic Press, New York, 1978.

[32] R.P. Chhabra, Bubbles, Drops, and Particles in Non-Newtonian Fluids, CRC Press, Boca Raton, FL, 1993.

[33] G. McKinley, Transport Processes in Bubbles, Drops and Particles, second ed., Taylor \& Francis, New York, 2002.

[34] I. Machač, B. Šiška, R. Teichman, Chem. Eng. Proc. 41 (2002) 577-584. 